\title{
Root system traits impact early fire blight susceptibility in apple (Malus $\times$ domestica)
}

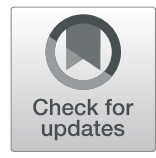

Jugpreet Singh"1, Jack Fabrizio ${ }^{1}$, Elsa Desnoues ${ }^{1}$, Julliany Pereira Silva ${ }^{1}$, Wolfgang Busch ${ }^{2}$ and Awais Khan ${ }^{1 *}$ (D)

\begin{abstract}
Background: Although it is known that resistant rootstocks facilitate management of fire blight disease, incited by Erwinia amylovora, the role of rootstock root traits in providing systemic defense against $E$. amylovora is unclear. In this study, the hypothesis that rootstocks of higher root vigor provide higher tolerance to fire blight infection in apples is tested. Several apple scion genotypes grafted onto a single rootstock genotype and non-grafted 'M.7' rootstocks of varying root vigor are used to assess phenotypic and molecular relationships between root traits of rootstocks and fire blight susceptibility of apple scion cultivars.

Results: It is observed that different root traits display significant $(p<0.05)$ negative correlations with fire blight susceptibility. In fact, root surface area partially dictates differential levels of fire blight susceptibility of 'M.7' rootstocks. Furthermore, contrasting changes in gene expression patterns of diverse molecular pathways accompany observed differences in levels of root-driven fire blight susceptibility. It is noted that a singular coexpression gene network consisting of genes from defense, carbohydrate metabolism, protein kinase activity, oxidation-reduction, and stress response pathways modulates root-dependent fire blight susceptibility in apple. In particular, WRKY75 and UDP-glycotransferase are singled-out as hub genes deserving of further detailed analysis.

Conclusions: It is proposed that low root mass may incite resource-limiting conditions to activate carbohydrate metabolic pathways, which reciprocally interact with plant immune system genes to elicit differential levels of fire blight susceptibility.

Keywords: Erwinia amylovora, Disease resistance, Root growth, Root mass, Root shoot interactions, Gene expression, Transcriptome, Co-expression network, Gene regulation, Grafting
\end{abstract}

\section{Background}

Roots play critical roles in plant function and their interactions with biotic and physical environments. Plant roots are increasingly recognized for their role in modulating systemic defenses of plants against pathogen infections via inter-organ signaling [1-4]. Roots can trigger physiological and genetic responses leading to activation of molecular pathways to recognize and resist pathogens upon infection [2-5]. Indeed, some root traits can act as physical barriers to soil-borne pathogens by hindering their penetration into living tissues [6-9]. Therefore, investigating interactions between roots and pathogens, as well as their relationships to disease susceptibility is of particular relevance to fruit tree crops wherein specific rootstocks

\footnotetext{
* Correspondence: awais.khan@cornell.edu

${ }^{1}$ Plant Pathology and Plant-Microbe Biology Section, Cornell University, Geneva, NY 14456, USA

Full list of author information is available at the end of the article
}

are frequently chosen to confer disease resistance for susceptible scion cultivars [10,11].

It has been reported that root system architecture (RSA) is dictated by growth, length, diameter, density, branching pattern, and branching angle of various root types, and it influences resource uptake from the soil [12-15]. In general, plant roots consist mainly of either one or more primary roots that, in-turn, produce several secondary and tertiary roots $[15,16]$. In contrast, the root system of apple rootstocks in commercial orchards consists mainly of adventitious roots originating from nodal junctions of stem cuttings, via vegetative propagation, which are important for initial establishment and success of grafted scions. Thus, growth and density of adventitious roots can influence the nutrient acquisition capacity of a plant, both under normal and stress prone conditions [17]. Nutrient uptake not only supports overall plant growth, but also contributes to plant survival under different stress 
conditions, such as wounding, flooding, drought, and nutrient deficiency [17-19]. However, the potential role of adventitious roots in enhancing tolerance to biotic and abiotic stresses remains unclear.

Rootstocks impact scion genotypes in many different ways. They can influence scion vigor and architecture, phenology, precocity, fruit quality, and production [20, 21]. In addition, rootstocks confer differential tolerance to salinity, drought, and disease-prone conditions in various crops [21-23]. For example, resistant rootstocks have been selected and used to enhance disease tolerance of grafted scion cultivars [11, 24-26] for sustainable disease management in commercial apple orchards. It has been proposed that rootstocks can modify scion phenotypes by altering levels of abscisic acid, cytokinin, auxin, and other hormones through long-distance signaling between roots and shoots [27-30]. Moreover, rootstock-regulated gene expression differences and mobile mRNA movements may also contribute towards enhanced host defense against pathogen infection [11, 25, 31]. For example, rootstocks influence expression levels of disease-associated genes of jasmonic acid and inositol pathways in grafted apple scions under fire blight infection [11]. These rootstock-derived mobile mRNAs may act as longdistance signals [31] to alter expression of disease-related molecular pathways in grafted scions. Roots also produce secondary metabolites, such as nicotine, furocoumarins, and aldehydes to improve plant defense mechanisms against pathogens [4]. In contrast, foliar bacterial infection alters secretion of malic acid in roots to recruit beneficial soil bacteria and improve plant immunity against pathogen attack [32]. Furthermore, it is likely that rootstocks may also influence scion physiology by regulating levels of water and nutrient uptake [22,33], which in turn can impose limits on pathogen spread and disease infection. Overall, root traits of rootstocks can play critical functional roles in regulating above-ground plant physiology and disease susceptibility of scions.

Fire blight, a systemic bacterial disease incited by Erwinia amylovora (Burr.) [34] causes extensive apple production losses worldwide. Fire blight infection can occur at multiple stages of plant development with higher risks of infection occurring particularly in new growing tissues of young orchards $[35,36]$. Apple growers mainly rely on use of chemical treatments and of pruning of infected twigs to control fire blight in commercial orchards, but these preventive control measures remain inefficient once bacteria have already invaded reproductive and/or vegetative plant tissues. Plant resistance provides alternative options for sustainable control of bacterial spread, particularly once bacteria penetrate host tissues.

Use of resistant rootstocks serves to directly manage fire blight infection of rootstocks, but it can also limit its spread to susceptible scions $[26,36]$. For example, susceptible scion cultivars grafted onto G.16, G.30, and G.11 apple rootstocks from the Geneva, New York apple rootstock breeding program have demonstrated high to moderate levels of resistance against fire blight $[26,37]$. This observed rootstock-driven differential fire blight resistance of grafted scions is attributed to changes in gene expression of disease-related proteins and pathways, including those of phytohormones, transcription and signal transduction activities, as well as of various cellular and metabolic responses [11]. Rootstocks can potentially utilize several mechanisms to confer resistance to scions, but the precise mechanism of rootstock-defined scion resistance or tolerance to fire blight remains unknown.

In this study, we have tested the hypothesis that apple rootstocks of higher root mass $(\mathrm{g})$ can respond more effectively to fire blight infection. To pursue this, the following two experiments have been conducted. In one experiment, a range of apple scion genotypes are grafted onto a single apple rootstock, 'Malling 7' ('M.7'), grafted trees are allowed to grow, and are then challenged with artificial inoculation with E. amylovora to establish the relationship between root vigor and disease severity in variable genetic backgrounds. In the second experiment, non-grafted 'M.7' rootstocks of varying root mass are grown, and then these are challenged with E. amylovora to specifically test the effect of root vigor on disease severity in a single genotype. Morphological characteristics were evaluated in both the experiments that led to assess molecular interactions between root traits of rootstock and fire blight susceptibility of apples.

\section{Results}

\section{Rootstock root traits and fire blight susceptibility of grafted scions are correlated}

Data from 45 grafted scions on M.7 rootstocks (Additional file 9: Table S1) were used to evaluate the relationships between root, shoot, leaf, and fire blight infection severity traits. Both root dry mass (g) and average roots per node (count) showed a wide range of variation in this population of scion genotypes. The root dry mass of M.7 rootstocks varied from $0.67 \mathrm{~g}(\mathrm{~g})$ to $6.84 \mathrm{~g}$ (Additional file 1: Figure S1A), whereas average number of roots per node ranged from 2.96 to 7.56 in M.7 rootstocks (Additional file 1: Figure S1B). Furthermore, corresponding shoot and leaf traits also showed significant $(p<0.05)$ variations in this population (Additional file 1: Figure S1C-E). Specifically, shoot and leaf lengths varied from 8.8 to $24.5 \mathrm{~cm}$ and 3.0 to $6.6 \mathrm{~cm}$ in this population, respectively, while SPAD values for leaf chlorophyll measurements ranged from 28.4 to 38.5. Moreover, shoot traits showed moderate broad-sense heritability $\left(\mathrm{H}^{2}\right)$ values, ranging from 0.70 to 0.62 , for leaf length and for leaf chlorophyll content. Of particular interest, percent lesion length ranged from 1.2 to $93 \%$ and showed 
significant $(p<0.05)$ variation in the population (Additional file 1: Figure S1F). The $\mathrm{H}^{2}$ values for percent lesion length was 0.71 .

To specifically examine the relationships between root dry mass and fire blight percent lesion length, we performed a statistical analysis of root dry mass (g) by dividing the 45 scion genotypes into three classes based on percent lesion length (Fig. 1). The clustering of scion genotypes led to increased numbers of replications per disease severity class for more robust statistical analysis. A significant $(p<0.05)$ difference was observed in the root dry mass (g) between three disease severity classes (Fig. 1). Similar results were obtained after removing the 20 scion genotypes displaying high standard deviation in percent lesion length (Additional file 9: Table S1).

Pairwise phenotypic correlations showed positive correlations between root and shoot traits, and negative correlations between root and fire blight susceptibility traits (Additional file 10: Table S2). However, not all correlations were significant. For instance, root dry mass (g) had significant $(p<0.05)$ negative correlation of -0.45 with percent lesion length (Additional file 2: Figure S2), whereas negative correlations between average roots per nodes and fire blight susceptibility traits were not significant. Similarly, root dry mass (g) displayed significant $(p<0.05)$ positive correlations with leaf length, but not with shoot length (Additional file 10: Table S2). Overall, these phenotypic correlations suggested that root dry mass of the rootstock could influence leaf growth and fire blight susceptibility of grafted scions.

Hierarchical clustering and multivariate analysis were used to categorize the entire population into groups based on their phenotypic differences. Therefore, this population was divided into six main clusters exhibiting distinct trait variation patterns, as illustrated in the heat map of traits values (Additional file 3: Figure S3). Furthermore, PCA of root and fire blight disease traits also highlighted phenotypic differences of these genotype clusters in this population (Fig. 2a). For example, root dry mass (g) and percent lesion length (\%) showed variable distribution patterns of trait means among the six identified clusters (Fig. 2b and c). In fact, clusters "C4" and "C6" tended to consist of genotypes with relatively lower root mass $(\mathrm{g})$ and higher disease susceptibility. However, this observed pattern was less clearly demonstrated in the remaining clusters.

Interestingly, nine principal components (PCs) explained the total variation present in this population. PC1 explained a maximum of $32.6 \%$ of the total variation. Although all traits contributed towards $\mathrm{PC} 1$ variation, root and shoot traits had positive contributions, while fire blight disease susceptibility traits contributed negatively to PC1 variation (Additional file 4: Figure S4). This trend supported previously detected negative correlations between root and fire blight susceptibility traits. In addition, analysis of higher-order PCs revealed different levels of contributions from root, shoot, and disease

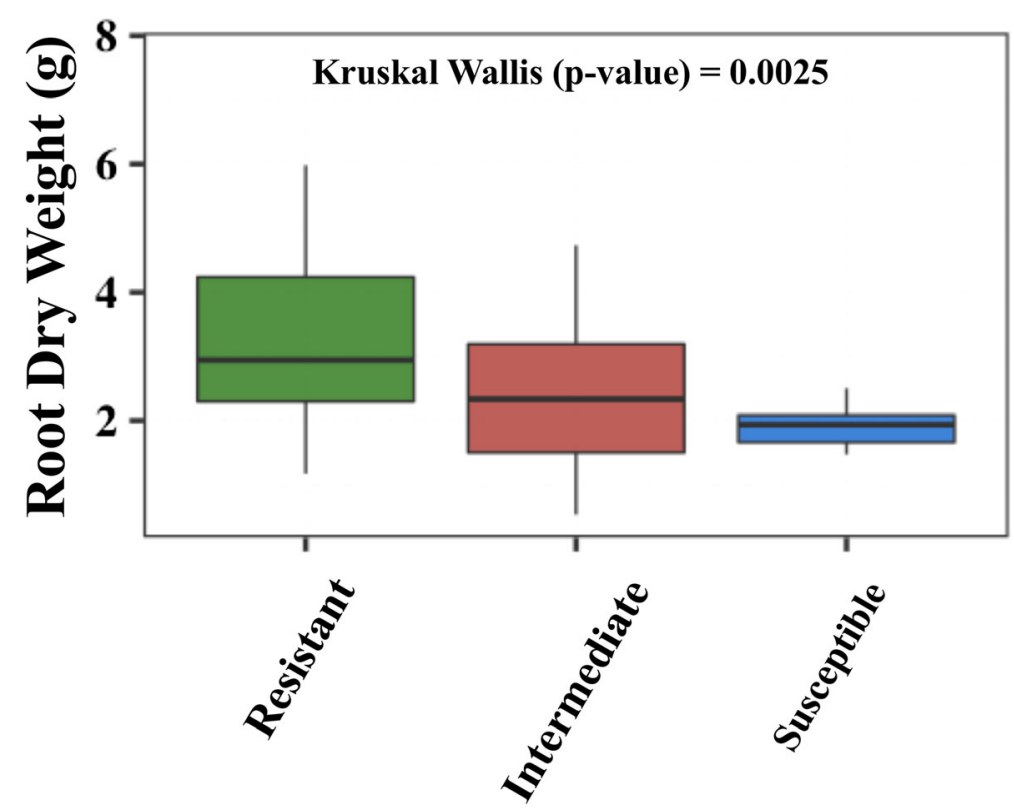

Fig. 1 Box plot showing distribution of root dry mass (g) in three disease severity classes observed in 45 grafted scion genotypes on 'M.7' rootstocks. The percent lesion length classes were defined as Resistant (0-20\% average PLL), Intermediate (21-80\% average PLL), and Susceptible (81-100\% average PLL). Error bars correspond to standard deviations of means 

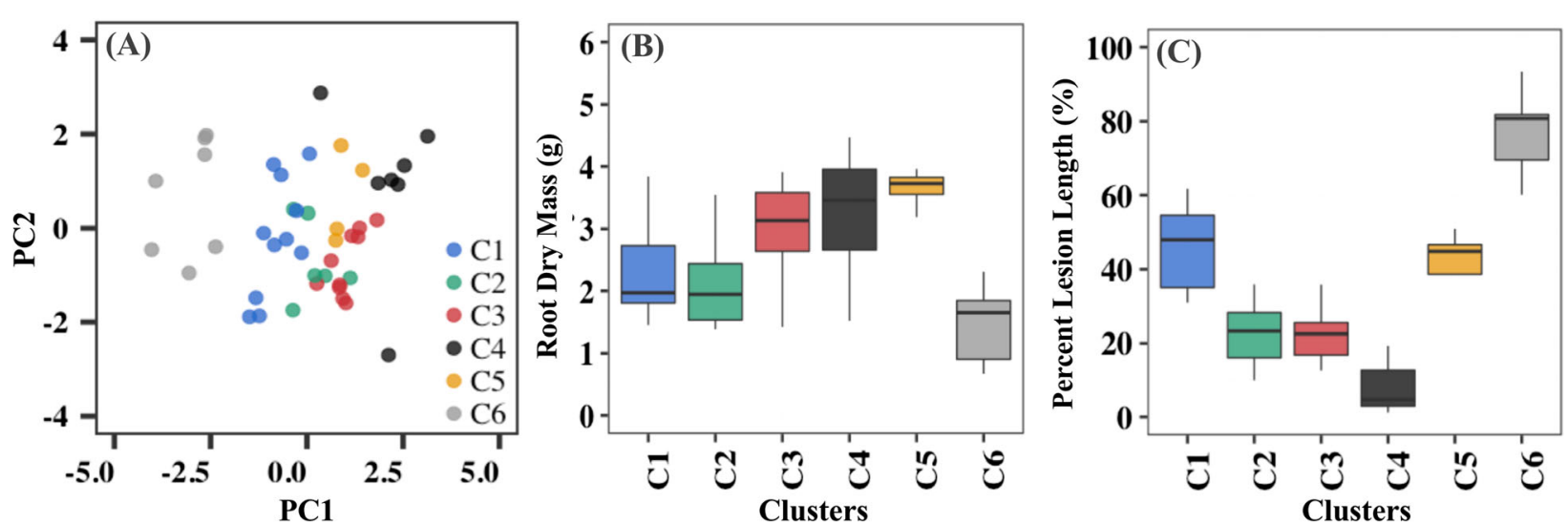

Fig. 2 Genotype clustering based on principal component analysis (PCA) and hierarchical clustering of root and disease traits. (a) Six genotype clusters obtained from a population of 45 scion genotypes grafted onto ' $M 7$ ' rootstocks. Distribution of means and variances in each cluster for (b) root dry mass (g) for C1-2.3, C2-2.2, C3-3.0, C4-3.8, C5-3.7, and C6-1.5; (c) percent lesion length (\%) for C1-45.9, C2-22.6, C3-22.4, C4-9.4, C5-40.5, and C6-76.7

susceptibility traits (Additional file 4: Figure S4), as noted by positive contributions from all root and disease-related traits to the PC2 variation. Overall, the PCA analysis revealed presence of considerable variation in this population, and this was partially driven by identified correlations between root growth and disease susceptibility traits.

\section{Rootstocks exceeding a root area threshold are less susceptible to fire blight}

To evaluate the extent to which roots can influence levels of fire blight susceptibility, a second independent experiment was conducted using non-grafted M.7 rootstocks representing four distinct root area classes (RACs). It was found that average root surface areas ranged from approximately 1720 (lowest RAC-1) to $4455 \mathrm{~cm}^{2}$ (highest RAC-4), corresponding to about 1.27 to 2.59 -fold change between the lowest and the other three RACs (Fig. 3a). Moreover, fire blight infection, measured as percent lesion length (\%), showed significant $(p<0.05)$ variations among the different RACs over time (Additional file 5: Figure S5). It was observed that absolute rates of disease progression from 2 to 8 dai were about 41.9 to $75.0 \%$ in RAC- 4 and RAC-3; whereas, these were higher, 84.4 to $98.3 \%$, in RAC-1 and RAC-2, respectively. At 8 dai, percent lesion length was significantly $(p<0.05)$ different in RAC-1 from those of RAC-3 and RAC-4, while this was not significantly different, at $p<0.05$, in RAC-2 from those of the other RACs (Fig. 3b). Overall, total infection and progression of disease were comparatively less in root classes of high root surface areas $\left(\mathrm{cm}^{2}\right)$ at the start of the experiment, and vice-versa. Moreover, the highest fire blight susceptibility was observed in rootstocks with a threshold of root surface area of $3644 \mathrm{~cm}^{2}$, represented by RAC-3 (Fig. 3).
Analysis of phenotypic correlations showed a strong correlation $\left(\mathrm{r}^{2}=0.87 ; p<0.05\right)$ of root areas $\left(\mathrm{cm}^{2}\right)$ between pre- and post-planting (and following bacterial inoculation), for a total duration of 106 days of growth, thus indicating that initial root area could serve as a predictor of root area growth at later stages of root development. Similarly, other root traits also demonstrated significant $(p<$ 0.05) positive correlations (Additional file 11: Table S3). For instance, root dry mass (g) showed high positive correlations with pre- and post-plant root area $\left(\mathrm{r}^{2}=0.82\right.$ and 0.93 , respectively). Similarly, both coarse and fine root mass showed significantly $(p<0.05)$ high positive correlations with root area $\left(\mathrm{cm}^{2}\right)$ before $\left(\mathrm{r}^{2}=0.79\right.$ and 0.62 , respectively) and after planting $\left(\mathrm{r}^{2}=0.85\right.$ and 0.78 , respectively). Some root traits also displayed significant $(p<$ $0.05)$ negative correlations with fire blight susceptibility traits. For instance, pre-plant root area $\left(\mathrm{cm}^{2}\right)$ and fine root dry mass (g) had significant negative correlations of -0.70 and -0.58 with percent fire blight lesion length, respectively (Additional file 11: Table S3). In contrast, negative correlations of percent lesion length against post-planting root area $\left(\mathrm{cm}^{2}\right)$, coarse root dry mass $(\mathrm{g})$, and total dry mass (g) were not significant.

\section{Contrasting expression patterns of distinct sets of genes are associated with root-dependent fire blight susceptibility}

Following bacterial inoculation, phenotypic analysis identified significant $(p<0.05)$ differences in fire blight infection over time in leaves of M.7 rootstocks belonging to different RACs (Fig. 3; Additional file 5: Figure S5). To identify molecular changes related to root-regulated fire blight susceptibility, gene expression patterns were characterized in leaf tissues of contrasting root area classes (RAC-1 with an average root area of $1720 \mathrm{~cm}^{2}$ vs. 


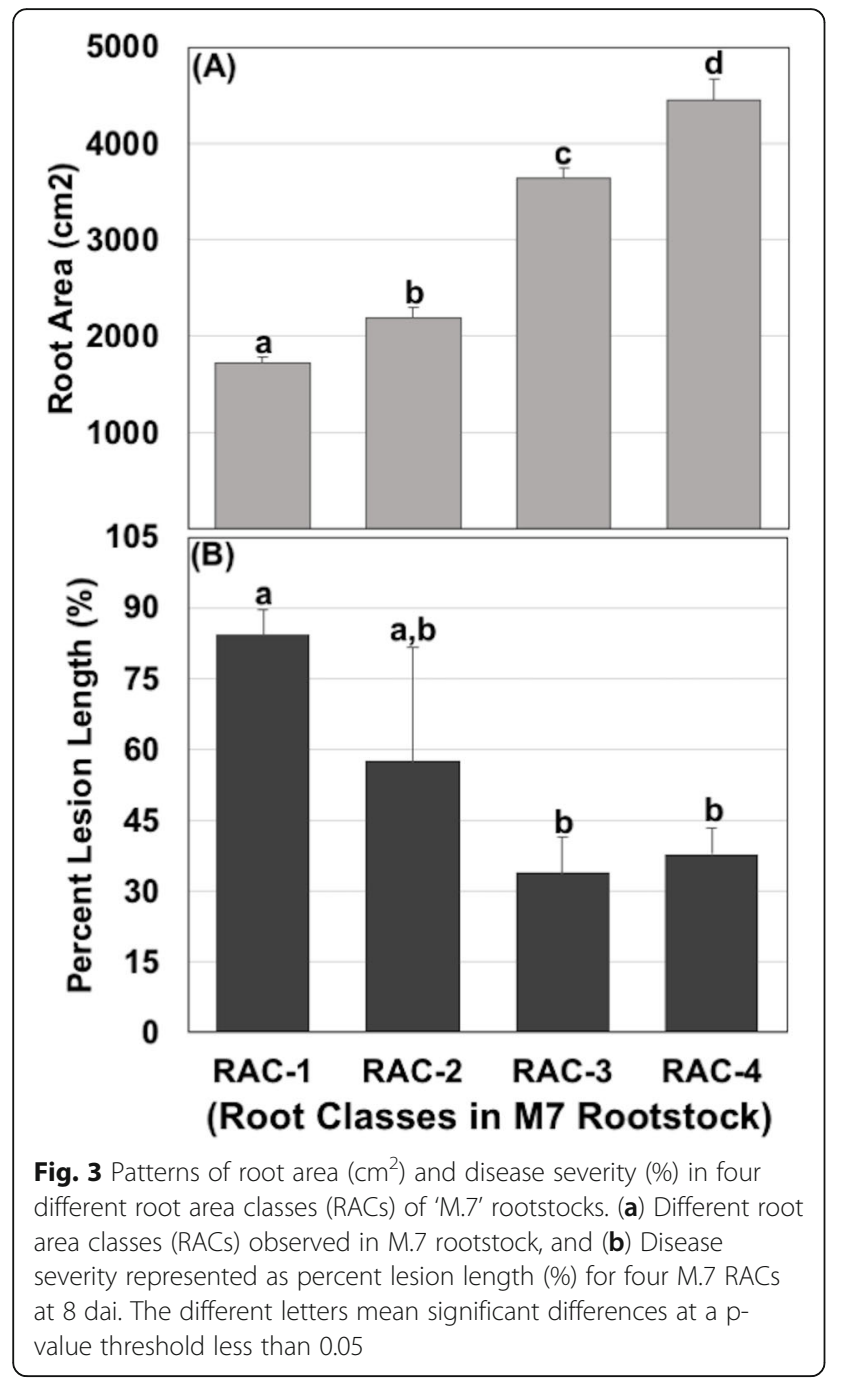

RAC-4 with an average root area of $4455 \mathrm{~cm}^{2}$ ) of nongrafted M.7 genotypes under control and bacterial inoculation treatments, at 4 and 8 dai (Additional file 12: Table S4). This gene expression analysis was conducted in sequential steps (Fig. 4). We first compared control leaf samples between RAC-1 and RAC-4 to identify any differentially expressed genes (DEGs) accounting for effects of root surface area differences on leaf responses. Next, control and bacterial-inoculated leaf samples were analyzed over time to identify fire blight responsive genes in leaf tissues. As a result, a set of common genes from both analyses were identified and deemed as genes associated with root-regulated fire blight susceptibility responses in leaf tissues.

Subsequently, it was observed that a high $\mathrm{PC} 1$ variation (82\%) was present in this population (Additional file 13), thus suggesting that root area, fire blight infection, and sampling time contributed to the variability detected in the gene expression dataset. Furthermore, the number of significant $(p<0.05)$ DEGs increased over time in both control and bacterial-inoculated RAC-4 samples, while these decreased in bacterial-inoculated RAC-1 samples over time (Fig. 4a). Interestingly, a total of 132 and 1017 DEGs were detected between RAC- 1 and RAC- 4 at 4 and 8 dpi, respectively (Table 1; Additional file 14). All 132 DEGs at 4 dpi were also identified at 8 dpi (Additional file 6: Figure S6), which indicated that the effects of root surface area differences persisted, and in fact they became more severe over this developmental period. In addition, a set of 454 out of 1017 DEGs showed significant $(p<0.05)$ changes in transcript levels following bacterial infection (Fig. 4b; Additional file 15). These DEGs involved genes likely related to bacterial infection, whose expression levels were also dependent on differences in root surface areas, as noted in contrasting RACs, and referred to herein as root-regulated fire blight responsive (RRFBR) genes.

An analysis of normalized expression for RRFBR genes identified opposite trends in contrasting RACs at both 4 and 8 dai. For instance, about 31.7\% $(n=144)$ DEGs demonstrated increased expression levels in RAC-1, but decreased expression levels in RAC-4 following bacterial inoculation at both sampling times (Additional file 16). Likewise, 11.4\% ( $n=53)$ DEGs demonstrated decreased expression levels in RAC-1, but increased expression levels in RAC-4. Interestingly, only a few genes $(n=9)$ demonstrated similar patterns of changes in expression levels between two these RACs following bacterial infection (Additional file 16). These findings suggested that for the majority of RRFBR genes, differences in fire blight susceptibility between RAC- 1 and RAC-4 were mostly associated with contrasting gene expression patterns.

\section{Interactions between genes from multiple pathways accompany root-dependent fire blight susceptibility} Upon further gene ontology (GO) analysis, it was noted that $\sim 92 \%$ of RRFBR genes belonged to general stress response pathways related to metabolic response (42\%), catalytic activity (40\%), and oxidation-reduction (10\%) processes (Fig. 4c; Additional file 17), whereas $8 \%$ of RRFBR genes represented functional terms related to carbohydrate metabolic process, cell recognition, response to biotic stimulus, protein serine/threonine kinase activity, and apoplast (Fig. 4c; Additional file 17). Moreover, expression levels of DEGs varied within these pathways (Additional file 7: Figure S7). For example, some DEGs in carbohydrate metabolic and protein kinase pathways had lower levels of expression in RAC-1 than in RAC-4, while other DEGs displayed an opposite trend (Additional file 7: Figure S7). Interestingly, all six DEGs involved in defense response pathways demonstrated reduced levels of expression in RAC-1, but increased levels of expression in RAC-4 at 8 dai following 


\section{(A)}

No Fire Blight Treatment

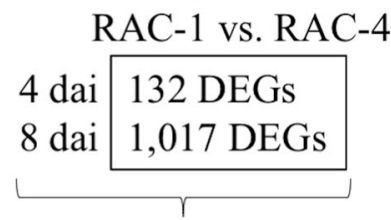

1,017 Unique DEGs
Control vs. Fire Blight

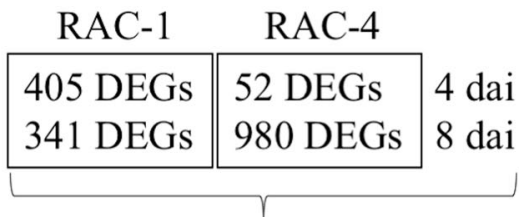

1,666 Unique DEGs

(B)

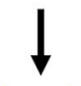

$$
\text { RACA IN:RACA }
$$

$\downarrow$

Control vs. Fire Blight

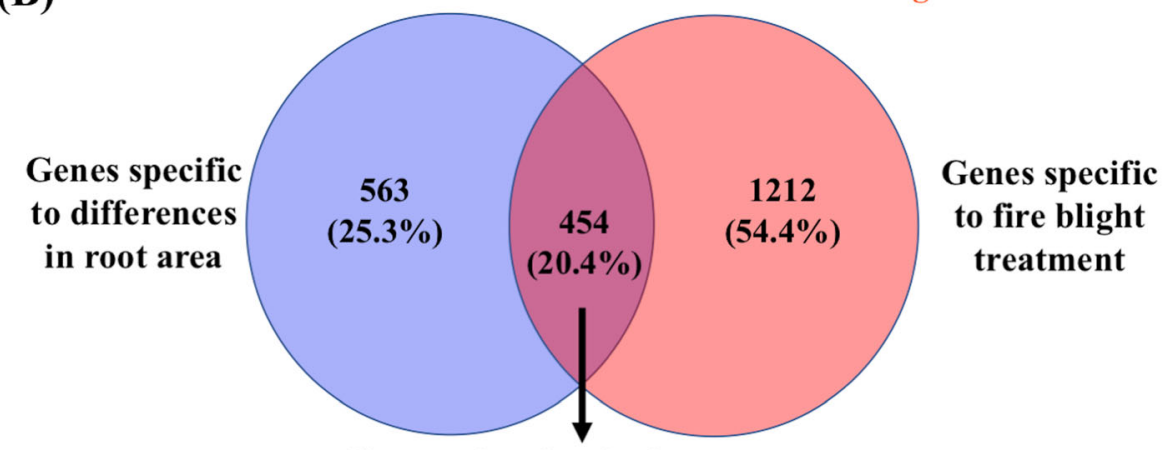

(C)

Genes related to both root area differences and fire blight infection

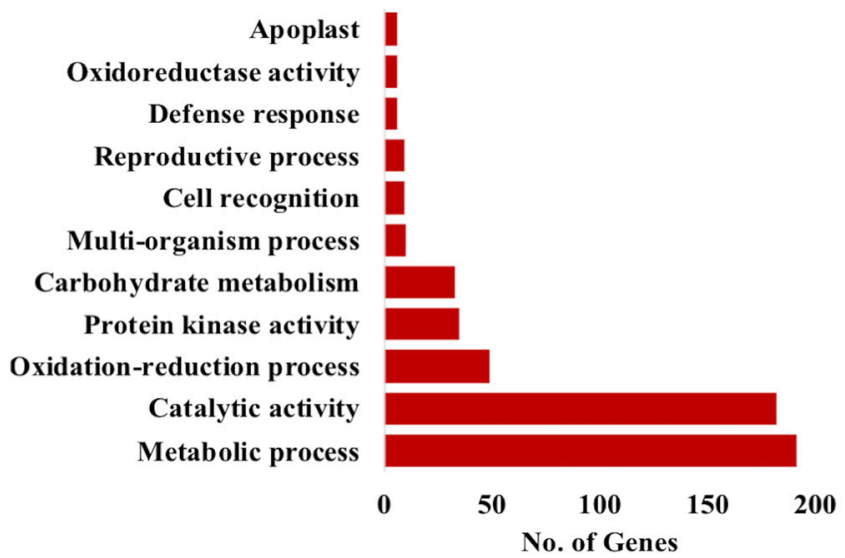

Fig. 4 A schematic representation of differential gene expression and pathway analysis for root-dependent fire blight infection in apple. (a) Numbers of differentially expressed genes (DEGs) obtained from analysis of control samples between RAC-1 and RAC-4, and between control and fire blight samples within RAC-1 and RAC-4. The DEGs were identified with a log2fold change of 1.5 between control and fire blight treated samples that also exhibit p-value of less than 0.01, (b) Venn diagram with numbers of unique and shared DEGs from two different expression analyses. (c) Pathways showing overrepresentation from common DEGs that most likely correspond to effects of low root area and fire blight infection

bacterial infection (Additional file 7: Figure S7; Additional file 17). Overall, these findings pointed toward likely interactions of both general stress response and carbohydrate metabolism pathways with defense-related genes. Indeed, these interactions would explain rootregulated differences in fire blight susceptibility in apple.

Subsequently, weighted co-expression analysis was used to identify co-expression patterns and putative interactions among RRFBR genes by identifying those hubs with the highest intramodular connectivity (Fig. 5a). It was found that a singular co-expression module " $\mathrm{C} 3$ " represented $77.3 \%$ of RRFBR genes (Additional file 8: Figure S8). In addition, UDP-glycosyltransferase, formate dehydrogenase, pathogenesis-related 4, WRKY DNAbinding protein 75, cysteine-rich RLK, cytochrome P450, laccase 7, glucose-methanol-choline oxidoreductase protein, and glycosyltransferase family proteins were found to be consistently present as highly connected genes in 
Table 1 Total number and percentage of differentially expressed (DE) genes for each comparison between two root area classes (RACs); lowest (RAC-1) and highest (RAC-4), and between control and fire blight (FB) infected samples at two time points after infection. The percentage of DE genes was calculated by comparing against total expressed genes $(n=35,224)$ in the transcriptome dataset. The genes were defined as DE based on the $p$-value $<0.01$ and log2Fold change of 1.5 from likelihood ratio test statistics using DESeq2. In the RAC-1 vs RAC-4 comparisons, the induced genes have comparatively higher gene expression in RAC-1, whereas repressed genes have comparatively higher gene expression in RAC-4

\begin{tabular}{|c|c|c|c|c|c|c|}
\hline Treatment & Time & Comparison & No. of DE Genes & DE Genes (\%) & Induced & Repressed \\
\hline \multirow[t]{2}{*}{ Root Area Classes } & 1 & RAC-1 vs RAC- 4 & 132 & 0.37 & 24 & 108 \\
\hline & 2 & RAC- 1 vs RAC- 4 & 1017 & 2.88 & 412 & 605 \\
\hline \multirow[t]{4}{*}{ Fire Blight Infection } & 1 & RAC-1 (Control vs FB Treatment) & 405 & 1.15 & 377 & 28 \\
\hline & 1 & RAC-4 (Control vs FB Treatment) & 52 & 0.15 & 39 & 13 \\
\hline & 2 & RAC-1 (Control vs FB Treatment) & 341 & 0.97 & 240 & 101 \\
\hline & 2 & RAC-4 (Control vs FB Treatment) & 980 & 2.78 & 549 & 431 \\
\hline
\end{tabular}

the "C3" module (Fig. 5b; Additional file 18). This suggested that interactions among core genes from the general stress response, carbohydrate metabolism, and defense pathways determined observed differences in fire blight susceptibility between RAC- 1 and RAC-4. In addition, detection of DNA-binding domain proteins as hubs in the "C3" module supported transcriptional regulation of co-expressed genes from these different pathways.

\section{Discussion}

Earlier studies have reported that rootstocks and rootstock system architecture influence various important traits of scion genotypes grafted onto these rootstocks $[20,21]$. As it has been demonstrated that rootstocks confer enhanced tolerance to salinity, drought, and disease in various crops [21-23], efforts have been undertaken to develop resistant rootstocks, which in turn can enhance disease tolerance of grafted scion cultivars $[11,24,25]$. In this study, it is observed that root traits of an apple rootstock (M.7), including root dry mass (g) and average roots per node (count), are indeed variable, and they do in turn influence shoot and leaf traits, including leaf chlorophyll contents, of different scion genotypes grafted onto this rootstock. More importantly, these root traits also influence response reactions of leaf and shoots of different scion genotypes to controlled inoculations with E. amylovora, and their susceptibility to fire blight disease. Although this latter finding confirms earlier reports [24, 26], it provides detailed analysis of the importance of root mass traits on fire blight reactions of grafted scion genotypes upon infection by $E$. amylovora. This finding is further supported by significant $(p<0.05)$ negative correlations obtained between root mass and scion fire blight susceptibility in these grafted apple trees.

Upon analysis of RACs of non-grafted 'M.7' rootstocks on fire blight susceptibility of above-ground leaf tissues,

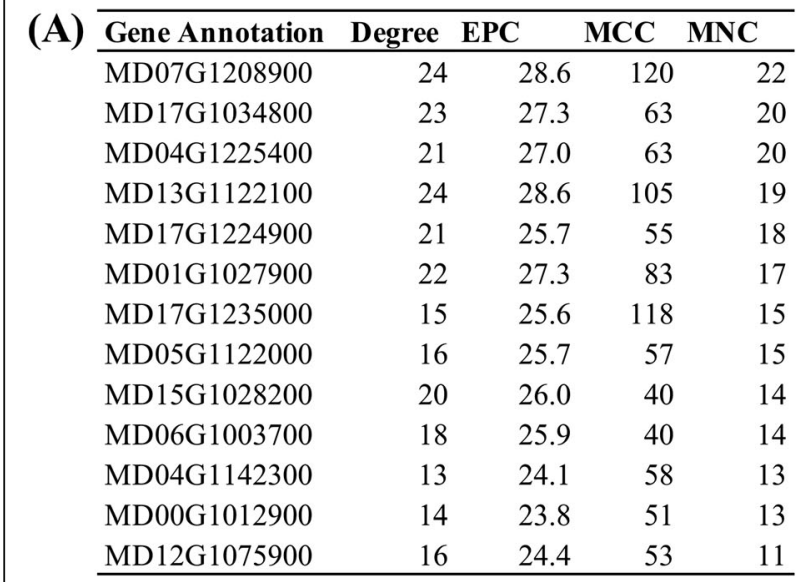

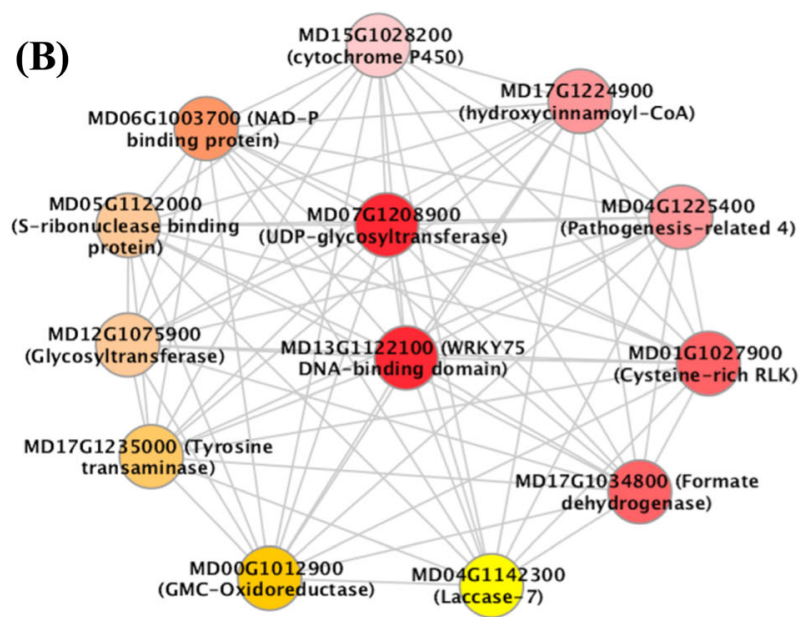

Fig. 5 Hub genes identified from weighted gene co-expression network analysis of differentially expressed genes (DEGs). (a) Detected hub genes and their corresponding network connectivity scores as measured by degree, EPC, MCC, and MNC algorithms, Chin et al. (2014). (b) Interconnected sub-module of these hub genes, wherein different colors represent connectivities from highest (red) to lowest (yellow) 
it is observed that increased root surface area contributed to decreased fire blight susceptibility in these above-ground leaf tissues, and the reverse is found to be true as well. These observed root-dependent differences in levels of fire blight disease susceptibility may be attributed to presence of multiple defense mechanisms $[11,25,38-40]$ that interact with the pathways related to nutrient status of a plant. Indeed, involvement of diverse molecular pathways related to plant metabolism, cell cycle, oxidation-reduction, and stress response suggests presence of systemic regulation of fire blight infection in apples [11, 38]. Moreover, rootstock genotypes can significantly contribute to scion tolerance to fire blight susceptibility via rootstock-regulated gene expression patterns [11].

It is important to note that different fire blight disease reactions and root traits have displayed significant $(p<$ $0.05)$ levels of variations in the two experiments conducted in this study. The observed variation in percent lesion length (\%) could be attributed, in part, to the different genetic backgrounds of grafted scion cultivars, which was supported by calculated moderate broadsense heritabilities of percent lesion length (\%). The grafted scions could also influence the root traits of 'M.7' rootstock, but the short time frame ( 3 months since grafting and 8 days post inoculation) of each experiment in this study makes it harder to notice such effects. However, the effect of scion genotypes cannot be fully excluded and could vary depending on the scion genotypes used. Nonetheless, the significant differences in root traits remains apparent in three different disease severity classes of mixed genetic backgrounds, which supports the observed relationships between root and disease severity traits. It has been reported that variations in root traits of rootstocks might contribute to phenotypic plasticity due to their exposure to different nutrient regimes, soil, and environmental conditions during their earlier growth in the clonal rootstock [15, 41, 42]. Phenotypic plasticity of different traits can vary among different genotypes [43-46]. Thus, identifying genes for root phenotypic plasticity would support efforts to breed for rootstocks with more uniform root traits. This, in turn, would contribute to enhanced resistance against $E$. amylovora infection, particularly for young grafted apple trees grown in orchards.

In this study, detection of various DEGs, including several disease-related and pathogenesis proteins, between contrasting RACs point to the critical role of the central immune system in conferring root-dependent fire blight susceptibility/resistance reactions. Earlier studies have reported that some of the disease-related CC-NBS-LRR proteins confer major resistance against fire blight in apples $[39,40]$. These results suggest that gene interactions between core defense pathways and system-level metabolic and stress-responsive pathways may regulate root-dependent fire blight susceptibility/resistance reactions in apple. Moreover, these pathways may operate in coordination with sugar and carbohydrate metabolic pathways, which have demonstrated overrepresentation in contrasting RACs investigated in this study. Thus, it is likely that low root mass alters sink activities of a plant, which in turn can modify expression patterns of carbohydrate metabolism genes. Furthermore, changes in carbohydrate metabolism can alter a plant's defense response through an inter-connected signaling network of metabolic and stress-responsive genes. For example, restriction of below-ground root growth can alter both development and carbohydrate metabolism of aboveground leaf tissues [47-49]. In addition, alteration in metabolite levels in a source leaf can determine the defense response against pathogen infection [50-52]. In fact, detection of a single co-expression module, "C3", consisting of carbohydrate metabolism and diseaserelated proteins further supports viability of such a model in apple. Furthermore, presence of interacting WRKY and ethylene responsive DNA-binding transcription factors suggest transcriptional co-regulation of these pathways. Thus, low root mass may lead to resourcelimiting conditions in the plant, thereby contributing to changes in gene expression of pathogenesis and diseaserelated proteins through carbohydrate metabolism pathways. It is these changes in expression in the central plant immune system that would then eventually determine fire blight susceptibility levels in apple. These results may add to the evidence that plants initiate a systemic response against fire blight after sensing the disease infection in inoculated scions, and transmit signals to the rootstock, which in turn contributes to disease tolerance/resistance through multiple mechanisms. However, rootstocks can influence the disease severity in grafted scions through multiple paths. For instance, rootstock-regulated gene expression, mobile RNA signaling between root-shoot, and nutrient-pathogen interaction as proposed in this study could be some of the factors contributing towards rootstock effects on grafted scions $[4,11,25,31]$.

It is important to point out that co-expression analysis conducted in this study has also highlighted those core genes with the highest intra-modular connectivity. In particular, the WRKY75 transcription factor and an UDPglycotransferase are the top two genes displaying highest levels of connectivity within this network. Thus, these two genes are deemed as worthy candidates for further studies to assess their potential roles in various biotic and abiotic stress conditions. Furthermore, it will be interesting to identify those factors contributing to root-dependent differences in levels of fire blight susceptibility/resistance, which requires a rigorous experimental and functional 
validation of few candidate genes and is yet beyond the scope of this manuscript.

The root system directly regulates amounts of nutrients and water uptake, which in turn influence growth, physiology, and metabolism of grafted scions [22, 33, 53]. As smaller root systems can limit availability of nutrients or impose partial stress conditions, this in turn can influence levels of disease susceptibility. For instance, water deficit is reported to increase plant susceptibility against fungal infections in different plant species $[54,55]$. This is partially attributed to altered expression of host $\mathrm{R}$ genes and/or of pathogen effectors [54]. In this study, contrasting expression patterns of several disease resistance genes and leucine rich repeats have been detected between RAC-1 and RAC-4, thus suggesting incidence of changes in plant immunity under low root surface areas. Similarly, nitrogen availability can affect disease severity levels in plants [53, 56]; however, the precise mechanism of nutrientdependent changes in disease susceptibility levels remains unknown. Therefore, further studies should be conducted to determine the role(s) of resource limiting conditions and those factors involved in differences in rootdependent responses to fire blight susceptibility/resistance reactions in above-ground plant tissues.

Future studies are also needed to obtain more accurate estimates and interpretation of the relationships between root traits and disease severity as high variation in disease severity between replicates of the same genotype may have introduced noise in our analyses. Since several factors can contribute towards the high variation within genotypes, selection of uniform shoots at same age and equal amount of bacterial inoculum is one simple way to reduce this variation to some extent. In addition, using an increased number of biological replicates might also help to lower such variation within genotypes. Another informative set of experiments will be to extend similar analyses beyond the experimental time frame of this study as the results from this study suggest that disease progresses at different rates in plants with low and high root surface area.

\section{Conclusions}

In summary, root traits can influence levels of fire blight susceptibility of apples. An optimum root area threshold is required to achieve the maximum tolerance against fire blight; however, high plasticity of root traits can hinder maintenance of such an optimal root system in apple rootstocks. Therefore, further studies should be conducted to identify genes or growth conditions that control root size, branching, and root phenotypic plasticity, as this new knowledge will assist in efforts to design more uniform root systems for optimum vigor of clonal apple rootstocks. In addition, manipulation of core regulatory genes of stress-responsive pathways can contribute to enhanced plant tolerance to abiotic and biotic stresses imposed by restricted root growth and disease infection.

\section{Methods}

Plant material and growth conditions

One-year-old apple rootstocks of 'Malling 7' ('M.7'), a moderately fire blight-susceptible rootstock, were purchased from Willamette Nurseries Inc. (Canby, OR), and used in two different experiments. 'M.7,' a commercially important apple rootstock, was originally selected from traditional French rootstocks, known as 'Doucin', at East Malling Research Station (UK).

To evaluate influence of root mass of rootstocks on fire blight susceptibility of grafted scions, 45 different scion genotypes were grafted onto 1-year-old 'M.7' rootstocks (Additional file 1: Table S1). Bud-wood of scion genotypes was collected from the US National Apple Collection that is maintained from a long time at USDA-ARS Plant Genetic Resources Unit (PGRU) located in Geneva, NY. These grafted trees were maintained in a moist dark chamber for a period of three months to promote healing of the graft unions. Then, these grafted trees were planted in $\mathrm{D} 40 \mathrm{H}$ deepots (Stuewe and Sons, Tangent, OR), $6.5 \mathrm{~cm}$ in diameter and $24.2 \mathrm{~cm}$ in depth, containing a standard Cornell soil mix (50 peatmoss:50 vermiculite with $6.2 \mathrm{~kg} \cdot \mathrm{m}^{-3}$ lime, $1.25 \mathrm{~kg} \cdot \mathrm{m}^{-3}$ superphosphate, and $0.62 \mathrm{~kg} \cdot \mathrm{m}^{3}$ calcium nitrate). These trees were allowed to acclimatize and grow in a greenhouse facility at Cornell AgriTech (Geneva, NY) maintained at $25^{\circ} \mathrm{C}, 50 \% \mathrm{RH}$, and $16 \mathrm{~h}$ light $/ 8 \mathrm{~h}$ dark photoperiod for a period of 8 weeks. For each scion genotype, three replications were maintained in the greenhouse facility at the Cornell AgriTech (Geneva, NY), and arranged in a completely randomized block design.

To assess the effects of varying root mass (g) on fire blight susceptibility, 21 non-grafted 'M.7' rootstocks were used in a second experiment. One-year-1-yearold 'M.7' rootstocks were pruned from the bottom up, using Fiskars hedge shears, to alter the numbers of adventitious root nodes growing along each of the rootstocks. The resulting rootstocks were photo-imaged, and analyzed using ImageJ (https://imagej.nih.gov/ij/) to identify four classes (RACs) that exhibit significantly different root area from one another. These nongrafted 'M.7' rootstocks were then potted in plastic pots $(26 \mathrm{~cm}$ in diameter and $22.5 \mathrm{~cm}$ in depth) using the Cornell Soil mix as described above. For each RAC rootstock treatment, three replications were used, and these trees were maintained in the greenhouse facility at Cornell AgriTech, arranged in a completely randomized block design, under conditions of $25^{\circ} \mathrm{C}, 50 \% \mathrm{RH}$, and $16 \mathrm{~h}$ light $/ 8 \mathrm{~h}$ dark photoperiod for a period of 106 days. 


\section{Fire blight inoculation and trait evaluation}

Bacterial inoculum was prepared using a highly virulent E. amylovora strain, Ea2002A obtained from Dr. Steve Beer's collection at Cornell University. Frozen inoculum stock was transferred to a petri plate containing King's B medium (KB), and incubated for $48 \mathrm{~h}$ at $28^{\circ} \mathrm{C}$. Bacterial cells were recovered in a suspension culture using $1 \mathrm{X}$ PBS, and adjusted to a concentration of $10^{9} \mathrm{CFU} / \mathrm{ml}$ on a SmartSpec Plus Spectrophotometer (Bio-Rad Laboratories, Hercules, CA, USA).

Potted young trees were inoculated with either bacteria (treatment) or water (control; only in second experiment) for fire blight evaluation. The youngest unfolded leaf of an actively growing shoot of a potted young tree was inoculated by bisecting across the midribs using scissors dipped in the bacterial suspension, as described earlier [38, 57]. Deionized water was used to bisect midribs of leaves of control plants in the second experiment.

All inoculated young trees were evaluated 8 days after inoculation (dai) for fire blight infection, and for root traits. For both experiments, total shoot length, total leaf length, and length of necrosis of a leaf were measured in ' $\mathrm{cm}$ ' using a ruler. The percent leaf lesion length (\%) was calculated as the ratio of necrotic lesion length of a leaf to total leaf length multiplied by 100 . Furthermore, chlorophyll contents of control and infected leaves were measured using a SPAD 502 Plus Chlorophyll Meter (Spectrum Technologies, Aurora, IL, USA). Average roots per node (count) and root dry mass (g) were simultaneously evaluated for all inoculated young trees (control and fire blight treated). Average roots per node were calculated by dividing total number of roots by number of nodes of a rootstock cutting used. At the end of each experiment at 8 dai, roots were shaved off each of the rootstocks, and dried in an oven to determine dry root mass.

For the second experiment, additional root trait data were digitally collected both at the beginning and at the end of the experiment. The root system from each 'M.7' rootstock was photographed by rotating it 360 degrees to capture the three-dimensional root surface area using a Canon EOS Rebel T5 Digital SLR camera (Cannon USA Inc., Melville, NY, USA). All raw images were first converted into greyscales, and then followed by binary conversion using the software ImageJ (https://imagej.nih. gov/ij/). Binary images were used to calculate the total root surface area $\left(\mathrm{cm}^{2}\right)$ at the beginning of the experiment. Rootstocks were categorized into four different RACs, from lowest to highest root surface area $\left(\mathrm{cm}^{2}\right)$. At the end of the experiment, roots were carefully dug out, and washed using a detergent and water. Roots were then spread on a flat surface, and photographed using a Canon EOS Rebel T5 Digital SLR camera. Photo-images were processed using an ImageJ software to calculate pre- and post-experiment root surface areas $\left(\mathrm{cm}^{2}\right)$. Based on digital root diameter classifications, the root system of each young tree was separated into coarse (diameter > $1 \mathrm{~mm}$ ) and fine (diameter $<1 \mathrm{~mm}$ ) roots, dried in an oven, and then used to determine fine root dry mass (g), coarse root dry mass (g), and total root dry mass (g).

\section{Statistical analysis}

All data collected for root and shoot traits, as well as for fire blight disease severity were used for statistical analysis. To test the relationships between root dry mass ( $\mathrm{g}$ ) and percent lesion length (\%), the genotypes were grouped into three categories based on percent lesion length as Resistant (0-20\% average PLL), Intermediate (21-80\% average PLL), and Susceptible (81-100\% average PLL). The data was tested for normality using Shapiro-Wilk test in R statistical software (http://www.R-project.org/) and log transformation was used to normalize the non-normal data. The normalized data was subjected to analysis of variance (ANOVA) using an R statistical software (http:// www.R-project.org/). In addition, we also performed Kruskal-Wallis test using the original non-normal dataset to observe the significant differences. Mean values were compared using Tukey's multiple comparison test. Broadsense heritability $\left(\mathrm{H}^{2}\right)$ was estimated as ratio of $V_{G} / V_{P}$, where $V_{\mathrm{P}}$ corresponded to the total phenotypic variance explained by the genetic component variance $\left(\mathrm{V}_{\mathrm{G}}\right)$. The absolute rate of disease progression was calculated as the difference in PLL between day 8 and day 2, divided by PLL at day 2.

Average trait values were used to calculate Pearson correlation coefficients, as well as to perform hierarchical clustering with the "hclust" function and a principal component analysis (PCA) using "prcomp" function in R (http://www.R-project.org/). Hierarchical clustering estimated individual relationships based on extent of similarities between them; whereas, PCA utilized variance components to determine such relationships. Trait mean values were scaled to conduct both hierarchical clustering and PCA analysis. For hierarchical clustering, scaled trait datasets were used to generate a Euclidean distance matrix for estimation of inter-cluster distance with Ward's linkage method. PCA analysis was conducted to obtain principal component (PC) eigenvalues and rotations to estimate contributions of different traits to explain variation by each PC. A PCA biplot was generated using the first two principal components (PC1 and $\mathrm{PC} 2$ ) to determine the overall genotypic variation and effects of root dry mass $(\mathrm{g})$ on disease severity.

\section{Leaf sample harvesting, RNA extraction, 3'RNAseq assay and sequencing}

Leaf tissues from M.7 rootstocks of contrasting initial root surface areas $\left(\mathrm{cm}^{2}\right)$, in the second experiment, were used for RNA extraction and for gene expression 
analysis. Leaves were collected at 4 and 8 dai from two biological replicates of control and three biological replicates of bacterial-inoculated young trees of two contrasting RACs as described earlier $[38,57]$. Leaf tissues were immediately immersed in liquid nitrogen, and stored at $-80^{\circ} \mathrm{C}$ until used for RNA extraction.

A SpectrumTM Plant Total RNA Kit (Sigma-Aldrich, St. Louis, MO, USA) was used to extract total RNA as per manufacturer's protocol. Leaf tissues were ground into fine powder in liquid nitrogen, and $100 \mathrm{mg}$ of leaf powder was transferred to $500 \mu \mathrm{l}$ of lysis solution containing $2 \% \beta$-mercaptaethanol. Samples were thoroughly mixed, placed at $56^{\circ} \mathrm{C}$ for $5 \mathrm{~min}$, and centrifuged for 1 min at $13,000 \mathrm{rpm}$. The supernatant was passed through a filtration column at $13,000 \mathrm{rpm}$ for $1 \mathrm{~min}$ to remove debris. The cleared lysate was mixed with $250 \mu$ l binding solution, and centrifuged through a binding column for $1 \mathrm{~min}$ at 13,000 rpm. After RNA binding, samples were washed twice using $500 \mu \mathrm{l}$ wash solution I, as per manufacturer's recommendations. Columns were centrifuged at maximum speed for $1 \mathrm{~min}$ during various washing steps. Dry columns were transferred to a new $2 \mathrm{ml}$ centrifuge tube, and $50 \mu \mathrm{l}$ elution buffer was added into the center of each binding column. Samples were kept in an elution buffer for $1 \mathrm{~min}$, centrifuged at a maximum speed for $30 \mathrm{~s}$ to elute RNA, and then this was repeated using $30 \mu \mathrm{l}$ of elution buffer to increase RNA yield. The amount of RNA was determined using a NanoDrop ${ }^{\text {tw }}$ Spectrophotometer (Thermo Fisher Scientific, Grand Island, NY, USA), and the quality of RNA samples was assessed by running a $1 \%$ bleach agarose gel.

A total of 20 libraries were constructed and sequenced for RNA samples from control and bacterial-inoculated samples at the Genomics Facility at Cornell University (Ithaca, NY, USA). Briefly, 3'RNAseq libraries were prepared from $\sim 500 \mathrm{ng}$ of total RNA per sample using the Lexogen QuantSeq 3' mRNA-Seq Library Prep Kit FWD for Illumina (https://www.lexogen.com/quantseq-3mrnasequencing/). Libraries were quantified on a Molecular Devices Spectra Max M2 plate reader (with the intercalating dye QuantiFluor), and pooled accordingly for maximum evenness. The pooled sample was quantified by digital PCR, and sequenced along a single lane of an Illumina NextSeq500 sequencer to obtain single-end $1 \times 86$ bp sequences. Pooled libraries were de-multiplexed based upon six-base i7 indices using an Illumina bcl2fastq2 software (version 2.17; Illumina, Inc., San Diego, CA, USA).

\section{Sequencing data processing and analysis}

A Trimmomatic (version 0.36) [58] software was used to remove Illumina adapters from de-multiplexed fastq sequences, as well as to remove low-quality reads for further analysis. Poly-A tails and poly-G stretches of at least 10 bases in length were then removed using the
BBDuk program in the package BBMap (https://sourceforge.net/projects/bbmap/), but keeping reads of at least 18 bases in length after trimming. Often, poly-G stretches are obtained from sequencing past ends of short fragments $(\mathrm{G}=$ no signal).

Trimmed reads were then aligned to the GDDH13 Version 1.1 apple genome assembly (https://iris.angers. inra.fr/gddh13/downloads/GDDH13_1-1_formatted.fasta. bz2) using the STAR aligner (version 2.5.3a) [59]. For the STAR indexing step, the gff3 annotation file (https://iris. angers.inra.fr/gddh13/downloads/gene_models_20170612. gff3.bz2) was converted into a gtf format the gffread program from cufflinks (version 2.2.1) [60]. Key parameters used in the STAR indexing step (--runMode genomeGenerate) include --genomeChrBinNbits 18 and --sjdbOverhang 100. The STAR alignment step used the following key parameters: --outReadsUnmapped Fastx, --outFilterMultimapNmax 10, --outFilterMismatchNoverLmax 0.06, --outSAMmode Full, --outSAMattributes Standard, --outFilterIntronMotifs, and RemoveNoncanonicalUnannotated. Output SAM files were converted to BAM using SAMtools (version 1.6) [61], and numbers of reads overlapping each gene in the gff3 file along the forward strand were counted using a HTSeq-count (version 0.6.1) [62]. A gene was deemed to be expressed using a criterion of detecting a minimum of five aligned high-quality read sequences against a particular gene model.

\section{Gene expression and enrichment analysis}

The R package DESeq2 (version 1.20.0) [63] was used to obtain normalized counts from raw read counts. These counts were then used to conduct PCA of the 500 most variably-expressed genes following count normalization and variance stabilizing transformation, as well as for differential gene expression analysis. Control and bacterialinoculated samples of each root class and time points were compared by deeming root class, time point, and inoculation treatment as distinct factors. The "contrast" function in DESeq2 was used to obtain expression analysis output for each comparison. For each gene, statistical significance of differential expression was based on a Wald test for a non-zero log fold change (LFC) estimate obtained from fitting a negative binomial generalized linear model [63]. Adjustment of $p$-values for multiple testing followed the Benjamini and Hochberg method [64]. Genes were deemed differentially expressed based on a log2Fold change threshold of 1.5 and a $p$-value of less than 0.01 . All upregulated genes were determined based on positive $\log 2$ Fold change values, and vice-versa.

Sets of differentially expressed genes from individual comparisons were used to perform a gene ontology (GO) term enrichment analysis using Fisher's exact test with agriGO v2.0 [65]. Differentially expressed genes (DEGs) were compared to the complete set of fully-annotated 
genes in the GDDH13 Version 1.1 apple genome assembly. Estimated $p$-values were corrected using the Hochberg false discovery rate (FDR) correction method in agriGO v2.0. A $p$-value cutoff of less than 0.05 was used to determine significantly enriched $\mathrm{GO}$ terms.

\section{Gene co-expression network analysis}

A co-expression network analysis of DE genes was performed using the weighted gene co-expression network analysis (WGCNA) package in R [66] to obtain modules of genes having similar expression patterns. A list of unique DE genes from each comparison was established by removing redundant genes from various differential gene expression analyses. Subsequently, normalized gene expression values for these unique DEGs were extracted to perform a co-expression network analysis. Co-expressed gene modules were then built using a single-step network generation and a module detection approach in WGCNA. Specifically, a network was generated by connecting all expression values in a dataset followed by detection of modules exhibiting very similar patterns of gene expression. A threshold value for module assignments was estimated using an unsigned topological overlap matrix (TOM); whereas, networks were constructed using a threshold power of 16 , branch cut height of 0.25 , and a minimum module size of 30. These co-expression modules were then visualized in Cytoscape v3.7.1 [67], and those highly connected genes, within each module, were identified using cytoHubba plugin [68] in Cytoscape. These modules were exported from WGCNA using a threshold of 0.2 , and the top 20 highly connected genes were identified using four different algorithms including MCC, MNC, Degree, and EPC, implemented in cytoHubba. Finally, outputs were compared to select those hub genes that were consistently detected from all the four algorithms.

\section{Supplementary information}

Supplementary information accompanies this paper at https://doi.org/10. 1186/s12870-019-2202-3.

Additional file 1: Figure S1. Boxplots showing variation in different root $(A-B)$, shoot (C-E), and fire blight infection (F) traits in 45 grafted scion genotypes on 'M.7' rootstocks.

Additional file 2: Figure S2. Pearson correlation coefficients $\left(R^{2}\right)$ of root dry mass ( $\mathrm{g}$ ) against percent lesion length (\%) of 45 grafted scion genotypes on 'M.7' rootstocks.

Additional file 3: Figure S3. Hierarchical genotype clustering of 45 grafted scion genotypes on 'M.7' rootstocks and cluster mean heatmap for different traits.

Additional file 4: Figure S4. Barplot showing rotations of first three principal components for different traits.

Additional file 5: Figure S5. Disease progression (percent lesion length) over time (2, 4, 6 and 8 dai).

Additional file 6: Figure S6. Volcano plots showing differentially expressed genes (DEGs) at 4 dai (A), and 8 dai (B). The unique and common DEGs are also shown in Venn Diagram (C).
Additional file 7: Figure S7. Heat map showing expression patterns of DEGs in protein kinase, carbohydrate metabolism, and defense pathways.

Additional file 8: Figure S8. Heat map showing expression patterns in three co-expression modules detected from weighted gene coexpression analysis.

Additional file 9: Table S1. List of genotypes used for analysis of relationships between root traits and disease severity.

Additional file 10: Table S2. Pairwise correlation coefficients between root, shoot, and disease traits in 45 grafted scions on 'M.7' rootstocks.

Additional file 11: Table S3. Pairwise correlation coefficients between root, shoot, and disease traits in different root area classes (RACs).

Additional file 12: Table S4. Summary of sequencing and read alignments against the apple genome assembly.

Additional file 13. Principal component analysis (PCA) using normalized read counts of apple genes.

Additional file 14. DEGs between RAC-1 and RAC-4 from control samples at 4 and 8 dai.

Additional file 15. DEGs between control and fire blight infected samples in RAC- 1 and RAC- 4 at 4 and 8 dai.

Additional file 16. Functional annotations and expression patterns of common 454 DEGs.

Additional file 17. Pathways showing enrichment in the common 454 DEGs.

Additional file 18. Hub genes in the " $\mathrm{C} 3$ " co-expression module.

\section{Abbreviations}

ANOVA: Analysis of Variance; BAM: Binary Alignment Map; CA: California; CCNBS-LRR: Coiled Coil Nucleotide Binding Site Leucine Rich Repeats; CFU: Colony-forming Unit; DEG: Differentially Expressed Gene; EPC: Edge Percolated Component; FDR: False Discovery Rate; GDDH: Golden Delicious Double Haploid; GO: Gene Ontology; IL: Illinois; KB: King's B medium; LFC: Log Fold Change; M.7: Malling 7; MCC: Maximal Clique Centrality; MNC: Maximum Neighborhood Component; NCBI: National Center for Biotechnology Information; NY: New York; OR: Oregon; PBS: Phosphatebuffered Saline; PC: Principal Component; PCA: Principal Component Analysis; PCR: Polymerase Chain Reaction; PGRU: Plant Genetic Resources Unit; PLL: Percent Lesion Length; RAC: Root Area Class; RH: Relative Humidity; RRFBR: Root-regulated Fire Blight Responsive; RSA: Root System Architecture; SAM: Sequence Alignment Map; SPAD: Soil Plant Analysis Development; SRA: Sequence Read Archive; STAR: Spliced Transcripts Alignment to a Reference; TOM: Topological Overlap Matrix; UK: United Kingdom; US: United States; WGCNA: Weighted Gene Co-expression Network Analysis

\section{Acknowledgments}

We thank Rebbeca Steiner for RNA extraction for sequencing.

\section{Authors' contributions}

AK designed the experiment. ED and JF conducted the first and second experiments respectively. JKS supported in the design and data collection of second experiment. JS performed the data analysis including gene expression, pathway enrichment and co-expression analysis. JS and AK interpreted the data and wrote the paper. WB supported the conceptualization and design of the experiments, interpretation of results and reviewing of the manuscript. All authors read and approved the manuscript.

\section{Funding}

This research was supported by the National Institute of Food and Agriculture, U.S. Department of Agriculture, Hatch project NYC-625410 and the Federal Capacity Funds Initiative managed by the New York State Agricultural Experiment Station (NYSAES), Cornell University, Geneva, New York, USA, and the NYSAES Director's Controlled Endowment Fund. This research aligns with the priorities of the funders but funders had no direct role in design of the study and collection, analysis, and interpretation of data or in writing the manuscript. 


\section{Availability of data and materials}

All read sequences from RNA-Seq analysis for all samples used in this study have been deposited in the National Center for Biotechnology Information (NCBI) sequence read archive (SRA) database under the Bioproject identifier PRJNA507638. The data supporting the conclusions of this research is provided as supplementary information.

\section{Ethics approval and consent to participate}

There are no ethical guidelines for research on bacterial Erwinia amylovora.

\section{Consent for publication}

Not applicable.

\section{Competing interests}

The authors declare that they have no competing interests.

\section{Author details}

'Plant Pathology and Plant-Microbe Biology Section, Cornell University, Geneva, NY 14456, USA. ${ }^{2}$ Salk Institute for Biological Studies, Plant Molecular and Cellular Biology Laboratory, and Integrative Biology Laboratory, $10010 \mathrm{~N}$ Torrey Pines Rd, La Jolla, CA 92037, USA.

Received: 4 June 2019 Accepted: 12 December 2019

Published online: 23 December 2019

\section{References}

1. Agut B, Gamir J, Jaques JA, Flors V. Systemic resistance to Tetranychus urticae induced by conspecifics is transmitted by grafting and mediated by mobile amino acids. J Exp Bot. 2016;67:5711-23.

2. Fragoso V, Rothe E, Baldwin IT, Kim S. Root jasmonic acid synthesis and perception regulate folivore-induced shoot metabolites and increase Nicotiana attenuata resistance. New Phytol. 2014;202:1335-45.

3. Nalam VJ, Keeretaweep J, Sarowar S, Shah J. Root-derived oxylipins promote green peach aphid performance on Arabidopsis foliage. Plant Cell. 2012;24:1643-53.

4. Erb M, Lenk C, Degenhardt J, Turlings TCJ. The underestimated role of roots in defense against leaf attackers. Trends Plant Sci. 2009;14:653-9.

5. Pieterse CMJ, Zamioudis C, Berendsen RL, Weller DM, Van Wees SCM, Bakker PAHM. Induced systemic resistance by beneficial microbes. Ann Rev Phytopathol. 2014;52:347-75.

6. Bani M, Perez-De-Luque A, Rubiales D, Rispail N. Physical and Chemical Barriers in Root Tissues Contribute to Quantitative Resistance to Fusarium oxysporum f sp pisi in Pea. Front Plant Sci. 2018:9:199.

7. Moura JC, Bonine CA, de Oliveira FVJ, Dornelas MC, Mazzafera P. Abiotic and biotic stresses and changes in the lignin content and composition in plants. J Integ Plant Biol. 2010;52:360-76.

8. Chisholm ST, Coaker G, Day B, Staskawicz BJ. Host-microbe interactions: shaping the evolution of the plant immune response. Cell. 2006;124:803-14.

9. Scherm $\mathrm{H}$, Coakley SM. Plant pathogens in a changing world. Austral Plant Pathol. 2003;32:157-65.

10. Koepke T, Dhingra A. Rootstock scion somatogenetic interactions in perennial composite plants. Plant Cell Rep. 2013;32:1321-37.

11. Jensen PJ, Halbrendt N, Fazio G, Makalowska I, Altman N, Praul C, Maximova SN, Ngugi HK, Crassweller RM, Travis JW, McNellis TW. Rootstock-regulated gene expression patterns associated with fire blight resistance in apple. BMC Genomics. 2012;13:9.

12. Khan MA, Gemenet DC, Villordon A. Root system architecture and abiotic stress tolerance: current knowledge in root and tuber crops. Front Plant Sci. 2016;7:1584

13. Rogers ED, Benfey PN. Regulation of plant root system architecture: implications for crop improvement. Curr Opin Biotech. 2015;32:93-8.

14. Wang $H$, Inukai $Y$, Yamauchi $A$. Root development and nutrient uptake. Crit Rev Plant Sci. 2006:25:279-301.

15. de Dorlodot S, Forster B, Pagès L, Price A, Tuberosa R, Draye X. Root system architecture: opportunities and constraints for genetic improvement of crops. Trends Plant Sci. 2007;12:474-81.

16. Pregitzer KS. Tree root architecture - form and function. New Phytol. 2008, 180:562-4.

17. Steffens B, Rasmussen A. The physiology of adventitious roots. Plant Physiol. 2016;170:603-17
18. Liao WB, Huang GB, Yu JH, Zhang ML. Nitric oxide and hydrogen peroxide alleviate drought stress in marigold explants and promote its adventitious root development. Plant Physiol Biochem. 2012;58:6-15.

19. Krauss KW, Allen JA, Cahoon DR. Differential rates of vertical accretion and elevation change among aerial root types in Micronesian mangrove forests. Estua Coas Shelf Sci. 2003:56:251-9.

20. Jensen PJ, Rytter J, Detwiler EA, Travis JW, McNellis TW. Rootstock effects on gene expression patterns in apple tree scions. Plant Mol Biol. 2003:493:493-511.

21. Warschefsky EJ, Klein LL, Frank MH, Chitwood DH, Londo JP, von Wettberg EJB, Miller AJ. Rootstocks: diversity, domestication, and impacts on shoot phenotypes. Trends Plant Sci. 2016;21:418-37.

22. Gautier AT, Chambaud C, Brocard L, Ollat N, Gambetta GA, Delrot S, Cookson SJ. Merging genotypes: graft union formation and scion-rootstock interactions. J Exp Bot. 2018:70:747-55.

23. Kumar P, Rouphael Y, Cardarelli M, Colla G. Vegetable grafting as a tool to improve drought resistance and water use efficiency. Front Plant Sci. 2017:8:1130

24. Zhu Y, Fazio G, Mazzola M. Elucidating the molecular responses of apple rootstock resistant to ARD pathogens: challenges and opportunities for development of genomics-assisted breeding tools. Hort Res. 2014;1:14043.

25. Jensen PJ, Makalowska I, Altman N, Fazio G, Praul C, Maximova SN, Crassweller RM, Travis JW, McNellis TW. Rootstock-regulated gene expression patterns in apple tree scions. Tree Genet Genom. 2010;6:57-72.

26. Norelli JL, Holleran HT, Johnson WC, Robinson TL, Aldwinckle HS. Resistance of Geneva and other apple rootstocks to Erwinia amylovora. Plant Dis. 2003;87:26-32.

27. Albacete A, Martínez-Andújar C, Ghanem ME, Acosta M, Sánchez-Bravo J, Asins MJ, Cuartero J, Lutts S, Dodd IC, Pérez-Alfocea F. Rootstock-mediated changes in xylem ionic and hormonal status are correlated with delayed leaf senescence, and increased leaf area and crop productivity in salinized tomato. Plant Cell Environ. 2009:32:928-38.

28. Albacete A, Martínez-Andújar C, Martínez-Pérez A, Thompson AJ, Dodd IC, Pérez-Alfocea F. Unravelling rootstockXscion interactions to improve food security. J Exp Bot. 2015;66:2211-26.

29. Tworkoski T, Fazio G. Hormone and growth interactions of scions and size-controlling rootstocks of young apple trees. Plant Growth Reg. 2016;78:105-19.

30. Venema JH, Giuffrida F, Paponov I, Albacete A, Perez-Alfocea F, Dodd IC. Rootstock-scion signalling: key factors mediating scion performance. In: Colla G, Perez Alfocea F, Schwarz D, editors. Vegetable grafting: principles and practices. Wallingford, UK: CABl; 2017. p. 94-131.

31. Yang $Y$, Mao L, Jittayasotorn $Y$, Kang Y, Jiao C, Fei Z, Zhong G. Messenger RNA exchange between scions and rootstocks in grafted grapevines. BMC Plant Biol. 2015:15:251

32. Rudrappa T, Czymmek KJ, Pare PW, Bais HP. Root-secreted malic acid recruits beneficial soil bacteria. Plant Physiol. 2008;148:1547-56.

33. Peccoux A, Loveys B, Zhu J, Gambetta GA, Delrot S, Vivin P, Schultz HR, Ollat $\mathrm{N}$, Dai Z. Dissecting the rootstock control of scion transpiration using model-assisted analyses in grapevine. Tree Physiol. 2018;38:1026-40.

34. Winslow CEA, Broadhurst J, Buchanan RE, Krumwiede C, Rogers LA, Smith $\mathrm{GH}$. 1920. The families and genera of bacteria. Final report of the Committee of the Society of American bacteriologists on characterization and classification of bacterial types. J Bacteriol. 1920;5:191-229.

35. Robinson T, Anderson L, Autio W, Barrit B, Cline J, Cowgill W, Crassweller R, Embree C, Ferree D, Garcia E, Greene G, Hampson C, Kosola K, Parker M, Perry R, Roper T, Warmund M. A multi-location comparison of Geneva 16, Geneva 41 and M.9 apple rootstocks across North America. Acta Hort. 2007; 732:59-65.

36. Russo NL, Robinson TL, Fazio G, Aldwinckle HS. Field evaluation of 64 apple rootstocks for orchard performance and fire blight resistance. HortScience. 2007:42:1517-25.

37. Norelli J, Aldwinckle H, Momol T, Johnson Bill, DeMarree A, Reddy MVB. Fire blight of apple rootstocks. New York Fruit Quarterly 2000; 8:2-5.

38. Silva KJP, Singh J, Bednarek R, Fei Z, Khan A. Differential gene regulatory pathways and co-expression networks associated with fire blight infection in apple (Malus $\times$ domestica). Hort Res. 2019;6:35.

39. Broggini GA, Wöhner T, Fahrentrapp J, Kost TD, Flachowsky H, Peil A, Hanke MV, Richter K, Patocchi A, Gessler C. Engineering fire blight resistance into the apple cultivar 'Gala' using the FB MR5 CC-NBS-LRR resistance gene of Malus $\times$ robusta 5. Plant Biotech J. 2014;12:728-33. 
40. Fahrentrapp J, Broggini GAL, Kellerhals M, Peil A, Richter K, Zini E, Gessler C. A candidate gene for fire blight resistance in Malus $\times$ robusta 5 is coding for a CC-NBS-LRR. Tree Genet Genom. 2013;9:237-51.

41. Ristova D, Busch W. Natural variation of root traits: from development to nutrient uptake. Plant Physiol. 2014;166:518-27.

42. Grossman JD, Rice KJ. Evolution of root plasticity responses to variation in soil nutrient distribution and concentration. Evol Appl. 2012;5:850-7.

43. Wu R, Grissom JE, McKeand SE, O'Malley DM. Phenotypic plasticity of fine root growth increases plant productivity in pine seedlings. BMC Ecol. 2004:4:14

44. Malamy JE. Intrinsic and environmental response pathways that regulate root system architecture. Plant Cell Environ. 2005;28:67-77.

45. Kadam NN, Tamilselvan A, Lawas LMF, Quinones C, Bahuguna RN, Thomson MJ, Dingkuhn M, Muthurajan R, Struik PC, Yin X, Jagadish SVK. Genetic control of plasticity in root morphology and anatomy of Rice in response to water deficit. Plant Physiol. 2017;174:2302-15.

46. Laitinen RAE, Nikoloski Z. Genetic basis of plasticity in plants. J Exp Bot. 2018;70:739-45.

47. Paul MJ, Pellny TK. Carbon metabolite feedback regulation of leaf photosynthesis and development. J Exp Bot. 2003;54:539-47.

48. Young IM, Montagu K, Conroy J, Bengough AG. Mechanical impedance of root growth directly reduces leaf elongation rates of cereals. New Phytol. 1997:135:613-9.

49. Robbins NS, Pharr DM. Effect of restricted root growth on carbohydrate metabolism and whole plant growth of Cucumis sativus L. Plant Physiol. 1988;87:409-13

50. Schwachtje J, Fischer A, Erban A, Kopka J. Primed primary metabolism in systemic leaves: a functional systems analysis. Sci Rep. 2018;8:216.

51. Lee DK, Ahn S, Cho HY, Yun HY, Park JH, Kwon SW. Metabolic response induced by parasitic plant-fungus interactions hinder amino sugar and nucleotide sugar metabolism in the host. Sci Rep. 2016;6:37434.

52. Ward JL, Forcat $S$, Beckmann M, Bennett M, Miller SJ, Baker JM, Hawkins ND, Vermeer CP, Lu C, Lin W, Truman WM, Beale MH, Draper J, Mansfield JW, Grant M. The metabolic transition during disease following infection of Arabidopsis thaliana by Pseudomonas syringae pv. Tomato. Plant J. 2010;63:443-57.

53. Fagard M, Launay A, Clement G, Courtial J, Dellagi A, Farjad M, Krapp A, Soulie M, Masclaux-Daubresse C. Nitrogen metabolism meets phytopathology. J Exp Bot. 2014;65:5643-56.

54. Bidzinski P, Ballini E, Ducasse A, Michel C, Zuluaga P, Genga A, Chiozzotto R, Morel J-B. Transcriptional basis of drought-induced susceptibility to the Rice blast fungus Magnaporthe oryzae. Front Plant Sci. 2016;7:1558.

55. Klutsch JG, Shamoun SF, Erbilgin N. Drought stress leads to systemic induced susceptibility to a nectrotrophic fungus associated with mountain pine beetle in Pinus banksiana seedlings. PLoS One. 2017;12:e0189203.

56. Moreau M, Degrave A, Vedel R, Bitton F, Patrit O, Renou JP, Barny MA, Fagard M. EDS1 contributes to nonhost resistance of Arabidopsis thaliana against Erwinia amylovora. Mol Plant-Microbe Inter. 2012;25:421-30.

57. Norelli JL, Farrell RE Jr, Bassett CL, Baldo AM, Lalli DA, Aldwinckle HS, Wisniewski ME. Rapid transcriptional response of apple to fire blight disease revealed by cDNA suppression subtractive hybridization analysis. Tree Genet Genom. 2009;5:27-40.

58. Bolger AM, Lohse M, Usadel B. Trimmomatic: a flexible trimmer for Illumina sequence data. Bioinformatics. 2014;30:2114-20.

59. Dobin A, Davis CA, Schlesinger F, Drenkow J, Zaleski C, Jha S, Batut P, Chaisson M, Gingeras TR. STAR: ultrafast universal RNA-seq aligner. Bioinformatics. 2013;29:15-21.

60. Trapnell C, Roberts A, Goff L, Pertea G, Kim D, Kelley DR, Pimentel H, Salzberg SL, Rinn JL, Pachter L. Differential gene and transcript expression analysis of RNA-seq experiments with TopHat and cufflinks. Nat Prot. 2012;7:562-78.

61. Li H, Handsaker B, Wysoker A, Fennell T, Ruan J, Homer N, Marth G, Abecasis $G$, Durbin R. 1000 genome project data processing subgroup. The sequence alignment/map (SAM) format and SAMtools. Bioinformatics. 2009;25:2078-9.

62. Anders S, Pyl PT, Huber W. HTSeq-a Python framework to work with highthroughput sequencing data. Bioinformatics. 2015;30:2114-20.

63. Love Ml, Huber W, Anders S. Moderated estimation of fold change and dispersion for RNA-seq data with DESeq2. Gen Biol. 2014;15:550.

64. Benjamini Y, Hochberg Y. Controlling the false discovery rate: a practical and powerful approach to multiple testing. J Royal Stat Soc Series B (Methodol). 1995;57:289-300.
65. Tian T, Liu Y, Yan H, You Q, Yi X, Du Z, Xu W, Su Z. agriGO v2.0: a GO analysis toolkit for the agricultural community, 2017 update. Nucl. Acids Res. 2017:45:W122-9.

66. Langfelder P, Horvath S. WGCNA: an R package for weighted correlation network analysis. BMC Bioinformatics. 2008;29:9-559.

67. Shannon P, Markiel A, Ozier O, Baliga NS, Wang JT, Ramage D, Amin N, Schwikowski B, Ideker T. Cytoscape: A software environment for integrated models of biomolecular interaction networks. Gen Res. 2003;13:2498-504.

68. Chin C, Chen S, Wu H, Ho C, Ko M, Lin C. CytoHubba: Identifying hub objects and sub-networks from complex interactome. BMC Sys Biol. 2014;8:S11.

\section{Publisher's Note}

Springer Nature remains neutral with regard to jurisdictional claims in published maps and institutional affiliations.
Ready to submit your research? Choose BMC and benefit from:

- fast, convenient online submission

- thorough peer review by experienced researchers in your field

- rapid publication on acceptance

- support for research data, including large and complex data types

- gold Open Access which fosters wider collaboration and increased citations

- maximum visibility for your research: over $100 \mathrm{M}$ website views per year

At BMC, research is always in progress.

Learn more biomedcentral.com/submissions 